\title{
Evolución y Perspectivas de la brecha digital en la Unión Europea
}

\section{Evolution and Perspectives of the digital divide in the European Union}

\section{Evolução e perspectivas do fosso digital na União Europeia}

\author{
Samaneh Kachouie ${ }^{1}$ \\ Universidad de Teherán (Irán) \\ kachouie.samaneh@ut.ac.ir \\ César Castilla ${ }^{2}$ \\ Universidad de Los Hemisferios (Ecuador) \\ cesarc@uhemisferios.edu.ec
}

Fecha de recepción: 06 de febrero de 2018

Fecha de recepción evaluador: 28 de septiembre de 2018

Fecha de recepción corrección: 12 de noviembre de 2018

\footnotetext{
${ }^{1}$ Samaneh Kachouie es candidata a un Ph. D en Gestión Medios por la Universidad de Teherán (Irán).

${ }^{2}$ César Castilla, Ph. D. es Profesor de relaciones internacionales centrado en la seguridad internacional, con especialización en temas de Medio Oriente (Irán) y Asia-Pacífico. Trabajo profesional y experiencia en investigación en Francia, Suecia, Alemania, Italia, China, Ecuador y Perú. Académico visitante en Think Tanks europeos como CERI (París 2015) ISDP (Estocolmo 2016), ZEI (Bonn 2017), IAI (Roma 2018). Miembro del grupo de trabajo de CLACSO sobre América Latina y Oriente Medio (2016-2019). Fundador y director del Programa de Integración para Refugiados y Migrantes en la Universidad de Los Hemisferios desde 2016. Profesor de la Maestría en Comunicación Digital de la Universidad de Los Hemisferios.
} 


\title{
Resumen
}

En la actualidad cada uno de los 28 miembros que componen la Unión Europea tienen diferentes indicadores en el Digital Economy and Society Index (DESI), el cual es un índice compuesto que resume los indicadores más relevantes con respecto al rendimiento digital en Europa y hace un seguimiento de la evolución de los estados miembros de la UE en materia de competitividad digital. Para hacer frente a esta problemática, se ha creado la Agenda Digital de la Comisión Europea que pretende disminuir considerablemente la brecha digital que afecta a los comunitarios en pleno siglo XXI. El presente artículo tiene como objetivo principal analizar los indicadores del DESI en función al Producto Bruto Interno (PIB) de los 5 países con el mayor y menor PIB dentro de la Unión Europea en el periodo 2014-2018. De esta manera, se podrá determinar los logros en materia de reducción de brecha digital en estos países; así como sus perspectivas para los próximos cinco años identificando los factores más importantes que permitan reducir considerablemente este problema.

Palabras clave: Brecha digital, Unión Europea, Inclusión Digital, Producto Interno Bruto, Índice digital socioeconómico

\begin{abstract}
Actually, the 28 members of the European Union have different results on the Digital Economy and Society Index (DESI), which is a composite index that summarizes the most relevant indicators on Europe's digital performance and digital competitiveness in order to understand the Digital Divide in this continent. To address this problem, the European Commission has created the Digital Agenda, which aims to reduce significantly the digital divide that affects Europeans in the XXI century. The main objective of this article is to analyze the indicators of the DESI according to the Gross Domestic Product (GDP) of the top five countries with the highest and lowest GDP within the European Union in the period 2014-2018. In this way, it will be possible to determine the achievements in challenges in reducing the digital divide in the EU; as well as the perspectives for the next five years identifying the most important factors that allow reduce considerably this problem.
\end{abstract}

Keywords: Digital divide, European Union, Digital Inclusion, Gross Domestic Product, Digital Economy and Society Index

\section{Resumo}

$\mathrm{Na}$ verdade, os 28 membros da União Européia têm resultados diferentes no Índice de Economia e Sociedade Digital (DESI), que é um índice composto que resume os indicadores mais relevantes sobre o desempenho digital e a competitividade digital da Europa para entender o Digital Divide neste continente. Para resolver este problema, a Comissão Europeia criou a Agenda Digital, que visa reduzir significativamente o fosso digital que afeta os europeus no século XXI. O principal objetivo deste artigo é analisar os indicadores do DESI de acordo com o Produto Interno Bruto (PIB) dos cinco principais países com o maior e o menor PIB da União Europeia no período 2014-2018. Desta 
forma, será possível determinar as conquistas nos desafios para reduzir o fosso digital na UE; bem como as perspectivas para os próximos cinco anos identificando os fatores mais importantes que permitem reduzir consideravelmente esse problema.

Palavras-chave: Digital divida, União Europeia, Inclusão Digital, Produto Interno Bruto, Economia Digital e Índice de Sociedade

\section{Introducción}

Hablar de la Unión Europea, es hacer un largo recuento que va desde el Tratado de París de 1951 hasta el Tratado de Lisboa 2007. Sin embargo, el primero representa la base para la conformación de la Comunidad Europea del Carbón y del Acero (CECA) cuyos países miembros eran Alemania, Bélgica, Francia, Italia, Luxemburgo y los Países Bajos. Esto se convertiría en un primer intento para fomentar la integración de los países europeos que desde una perspectiva liberal de las relaciones internacionales aseguraba la cooperación entre estados. Todo esto con la finalidad de alejar el fantasma de las Grandes Guerras y cimentar el camino hacia la paz que brinde estabilidad política y económica.

Con el transcurrir de las décadas, esta nueva forma de integración se fue volviendo más atractiva ya no solo para los países de Europa Occidental sino una vez que cayó el bloque socialista; lo fue también para los países de Europa Central y Oriental. En los años noventa, este proyecto de integración se convertiría en lo que conocemos en la actualidad como la Unión Europea, gracias a la firma del Tratado de Maastricht en febrero de 1992. A partir de este momento, el trabajo conjunto de los países que conformaban este bloque, apuntaba a trabajar de manera conjunta para conseguir una verdadera unión política y económica donde la cohesión social y territorial jugaban un rol fundamental. Un claro ejemplo de esto último, es el Euro que a inicios del siglo XXI se convierte en una moneda de curso legal para varios países cuya área de influencia se denominaría "Zona Euro" que en la actualidad está comprendida por 19 estados como Alemania, Austria, Bélgica, Chipre, Eslovaquia, Eslovenia, España, Estonia, Finlandia, Francia, Grecia, Irlanda, Italia, Letonia, Lituania, Luxemburgo, Malta, Países Bajos y Portugal.

Además, en el 2007 se firmaría el Tratado de Lisboa que entraría en vigencia a partir del 2009, cuyo objetivo principal era darle un mayor dinamismo a la Unión Europea no solo como proceso de integración sino también como una institución solida a nivel internacional. Con este último, se modificó el Tratado de Roma (1957) y el Tratado de Maastricht (1993).

En la actualidad, la Unión Europea cuenta con 28 miembros; a pesar del BREXIT, Reino Unido, sigue siendo miembro pleno con todos los derechos y obligaciones correspondientes (European Union, 2018). Si bien es cierto, la Unión Europea ha sido el proceso de integración más exitoso de la historia, al iniciar el siglo XXI ha tenido que enfrentar una serie de problemas y desafíos con los cuales tiene que luchar para poder seguir subsistiendo. Para estos fines, la Unión Europea se ha dotado de diferentes instituciones en las últimas décadas, siendo las más importantes el Parlamento Europeo, Consejo Europeo, Consejo de la Unión Europea, la Comisión Europea y el Servicio Europeo de Acción Exterior (SEAE). 


\section{Marco referencial}

Dentro de las metas y valores de la Unión Europea se ha planteado una serie de objetivos y valores donde la figura central es el individuo. Donde figuran como objetivos: promover la paz, sus valores y el bienestar de sus ciudadanos además de combatir la exclusión social y la discriminación; también incentivar el progreso científico y tecnológico; y valores como la igualdad (European Union, 2018). Esto implica que hay una predisposición por parte de este proceso de integración en mejorar el nivel de vida de sus ciudadanos, por lo cual el aspecto educativo en la Unión Europea es esencial para lograr sus objetivos. Dentro de este aspecto, se contempla la reducción de la Brecha Digital.

Tratar de definir lo que significa brecha digital, en la actualidad no es una cosa muy sencilla, dado al gran boom tecnológico que se ha venido desarrollando en el siglo XXI. Pascal Plantard en su libro titulado "Pour en finir avec la fracture numérique" comenta que este término aparece en algunos trabajos de investigación para describir la disparidad en el acceso al internet señalando a las minorías como es el caso de los afroamericanos en los Estados Unidos (Plantard, 2011, pág. 18). Por otro lado, la Dirección general de la Comisión Europea encargada de la información estadística "EUROSTAT" la define como

"la distinción entre aquellos que tienen acceso al mundo y aquellos que están excluidos de estos servicios. Contempla la participación de los ciudadanos y las empresas en la sociedad de la información la cual depende de las tecnologías de la información y la comunicación (TIC), es decir, la presencia de dispositivos electrónicos, como computadoras, y conexiones a Internet. El término también incluye el acceso a las TIC's, así como las habilidades relacionadas que se necesitan en la sociedad de la información. La brecha digital se puede clasificar de acuerdo con los siguientes criterios: edad, educación, ingresos, grupos sociales o ubicación geográfica" (Eurostat, 2016).

Más allá de una simple conceptualización, la brecha digital también puede ser analizada por las diferencias a través de la división norte-sur, donde se acostumbra a decir que el norte concentra la mayor cantidad de países desarrollados y el sur país en vías de desarrollo. Esto podría llevarnos a pensar que a mayor desarrollo económico la brecha digital sería menor y viceversa. En el aspecto geográfico, las ciudades por lo general van a tener mayor infraestructura que les permita tener mayor facilidad para el uso del wifi que las zonas rurales. Luego de esto podríamos también agregar que otro aspecto radica en la brecha generacional que está determinada por grupos etarios. Dado que los más jóvenes son los más proclives a usar dispositivos dotados de internet que aquellas personas que se encuentran en una edad avanzada, que por lo general rechazan lo digital.

Unión Europea en la actualidad cuenta principalmente con tres planes para reducir la brecha digital entre ellos tenemos, "Europe 2020 Strategy" (2010), Gigabit Society by 2025 (2016) y finalmente WiFi4EU (2017).

Con respecto a Europe 2020 Strategy está contemplado la reducción de la brecha digital a través de una Agenda Digital promovida por la Comisión Europea que constituye uno de los siete pilares de "Europe 2020 Strategy". Este plantea un crecimiento del empleo hacia el final de la década actual. Cabe resaltar que este crecimiento será 
inteligente, sostenible e integrador como una forma de superar las deficiencias estructurales en la economía europea enfocándose en aumentar el nivel de competitividad y productividad apuntandoa sustentar una economía social de mercado cuya principal característica será la sostenibilidad (European Commission, 2015). Dentro de este contexto, lo que se busca es aprovechar de la mejor manera el potencial de las Tecnologías de la Información y la Comunicación (TIC) con el objetivo de fomentar la innovación, el crecimiento económico y el progreso (European Commission, 2018).

Ante esta situación, Andrus Ansip, Vicepresidente y Comisario europeo de Mercado Único Digital explica que

"la digitalización de Europa está en marcha, pero muchos países deben intensificar sus esfuerzos. Todos los Estados miembros deben invertir más para aprovechar al máximo el mercado único digital. No queremos una Europa digital de dos velocidades. Debemos unir nuestros esfuerzos para hacer de la UE un líder mundial en tecnología digital" (European Commission, 2017).

El tráfico de Internet se encuentra expandiéndose en un $20 \%$ anual, e incluso más de un $40 \%$ por año en redes móviles. Lo importante es que el Parlamento Europeo y el Consejo están examinando de manera conjunta, las propuestas de la Comisión con el objetivo de reformar las normas de telecomunicaciones de la UE. Esto apunta a alentar la inversión en redes de muy alta capacidad para satisfacer las crecientes necesidades de conectividad de sus ciudadanos. Otra parte importante de este trabajo conjunto es determinar los objetivos estratégicos para una sociedad europea Gigabit para el 2025. Esta estrategia de la Comisión sobre conectividad para una sociedad europea Gigabit, fue adoptada en septiembre de 2016. Aquí se concibe una visión de Europa donde se prioriza la disponibilidad y la adopción de redes alta capacidad lo que permitirá el uso generalizado de productos, servicios y aplicaciones en el Mercado Digital Único Europeo. Esta visión tiene tres objetivos estratégicos para el 2025: Conectividad Gigabit para todos los principales impulsores socioeconómicos, cobertura ininterrumpida 5G para todas las áreas urbanas y principales rutas de transporte terrestre, y acceso a la conectividad que ofrece al menos $100 \mathrm{Mbps}$ para todos los hogares europeos (Euroepan Commission, 2018).

Pero lo más complicado de todo es que los Estados miembros deberían redoblar sus esfuerzos para alcanzar objetivos de una manera uniformizada en función a la asignación del espectro donde está previsto que se incluya una banda ya existente de 700 $\mathrm{MHz}$, para que las redes de comunicación de próxima generación (5G) puedan desplegarse ampliamente en 2020. Fundamentalmente, lo que resta hacer es trabajar en la coordinación del espectro de radio en territorio europeo, lo que se constituye en un elemento esencial para la cobertura inalámbrica. El aspecto financiero es importante, ya que los municipios del viejo continente podrán solicitar financiación para instalar wi-fi gratis en sus espacios públicos como parte del programa WiFi4EU de la Comisión (European Commission, 2018).

La propuesta aprobada por el Parlamento Europeo en mayo 2017 con respecto a "Wifi para Europa" (WiFi4EU), el cual en su momento inicial pretendía financiar wifi gratuito en lugares públicos como parques o bibliotecas hasta por un total de 120000 
millones de euros. Este proyecto sin duda alguna se convertirá en una idea trascendental teniendo como beneficiarios a aquellas personas de bajos recursos que no puedan acceder a la banda ancha o contar con servicio de internet desde sus hogares (Sander, La Tribune, 2017).

Con respecto a los progresos de la Unión Europea en los últimos años, el 72\% de sus ciudadanos usaban el internet al menos una vez por semana en el 2014. No obstante, el 20\% de ellos nunca habían usado el internet. Y en el caso de los ciudadanos europeos que, si lo usaban, el 62\% disponían de acceso a una banda ancha de 30Mbps. Por otro lado, esta cantidad contrasta con la del ámbito rural, donde al menos el 18\% solo tenía acceso a una banda ancha de 30Mbps. Esta situación, sin duda alguna se ha ido mejorando paulatinamente dado que la Unión Europea cuenta con algunas herramientas para reducir la brecha digital. Entre ellas los Fondos Europeos, Subvenciones monetarias directas, Prestamos con tasas bajas de interés, Políticas de compras públicas, Rebajas fiscales y Provisión de infraestructura pública (European Commission, 2014).

En marzo de 2017, el 76\% de los hogares europeos contaban con acceso a internet de muy alta velocidad, es decir al menos $30 \mathrm{Mbps}$; y en ciertos Estados miembros de la Unión Europea, mantienen una proporción significativa de estos hogares que ya pueden acceder a redes con la capacidad de entregar $100 \mathrm{Mbps}$. Por otro lado, más del $25 \%$ de los hogares en el viejo continente contaban con un servicio de banda ancha. Con respecto al número de suscripciones a servicios de plan de datos móviles, este aumentó de 58 suscriptores por cada 100 habitantes en 2013 a 84 en 2016.Lo que evidencia un avance importante en este rubro. Y finalmente, los servicios móviles $4 \mathrm{G}$ cubren el $84 \%$ de la población de la UE; Siendo esto bastante significativo, pero aún insuficiente para este bloque de integración. Lo que necesitan los países de la Unión Europea es satisfacer el aumento futuro en la necesidad de velocidad, calidad y confiabilidad de las conexiones (European Commission, 2017).

En junio 2017, tuvo lugar otro hecho que apunta a la reducción de la brecha digital que consiste en la abolición de las tarifas de roaming, algo tan esperado por los europeos desde hace una década que finalmente fue alcanzado. Por consiguiente, con esta disminución de los recargos telefónicos se espera un mayor volumen de conexiones a Internet móvil en cualquier lugar de Europa (European Union, 2017).

En el 2018, aún es posible decir que a pesar de todas las estrategias y los recursos que posee el proceso de integración europeo, todavía es fácil detectar algunos aspectos físicos, geográficos, sociales, financieros o regulatorios que se convierten en un impedimento que limitan la lucha en contra de la reducción de la brecha digital (Sander, La Tribune, 2017). Por ejemplo, en junio 2018, el 80\% de los europeos cuentan con acceso a Internet, la brecha digital está lejos de haber desaparecido. Y es que precisamente en este continente, continúa existiendo una gran proporción de "pobremente conectados" y más del 40\% de los ciudadanos europeos consideran que las habilidades digitales son nulas o sin importancia (Champain, 2018). 


\section{Metodología}

El presente artículo tiene como objetivo principal analizar los indicadores del DESI en función al Producto Bruto Interno (PIB) de los 5 países con el mayor y menor PIB dentro de la Unión Europea durante el periodo 2014-2018. Para lo cual se ha creído conveniente emplear el estudio de casos que comprenderá por un lado a los cinco países con el mayor PIB (Alemania, Reino Unido, Francia, Italia y España) y por el otro a los cinco países con el menor PIB (Letonia, Lituania, Eslovenia, Croacia, Bulgaria) (Datos Macro, 2018). A través de un análisis cualitativo, se pretende generar un mapeo de estos actores, roles y recursos con la finalidad de comprender la evolución y las perspectivas en la disminución de la brecha digital; no sin antes identificar los factores más importantes que permitan una disminución significativa de esta problemática para los próximos cinco años 2018-2022 en la Unión Europea.

\section{Hallazgos}

En el año 2017, la Unión Europea registró un PIB de 11.171.870M.€. De los 28 estados miembros de la Unión Europa, los cinco más importantes fueron: Alemania (3.263.350M.€), Reino Unido (2.324.293M.€), Francia (2.291.705M.€), Italia (1.716.935M.€) y España (1.163.662M.€) (Datos Macro, 2018). Por otro lado, los países con el PIB más bajo son Letonia (26.857M. €), Lituania (41.857M. €), Eslovenia (43.278M.€), Croacia (48.677M.€), Bulgaria (50.430M. €) (Datos Macro, 2018). No obstante, los países que aportan más al PIB de la UE no significa que hayan obtenido grandes logros en la disminución de la brecha digital y por el contrario los que menos aportan tampoco significa que estén tan mal posicionados en la disminución de la brecha digital. Esto se puede evidenciar según los resultados del Índice de Economía y Sociedad Digital (DESI), el cual agrupa 5 indicadores relevantes (conectividad, capital humano, uso de internet, integración de la tecnología digital y servicios públicos digitales) sobre el desempeño digital de Europa y rastrea la evolución de los estados miembros de la UE en competitividad digital (European Commission, 2018).

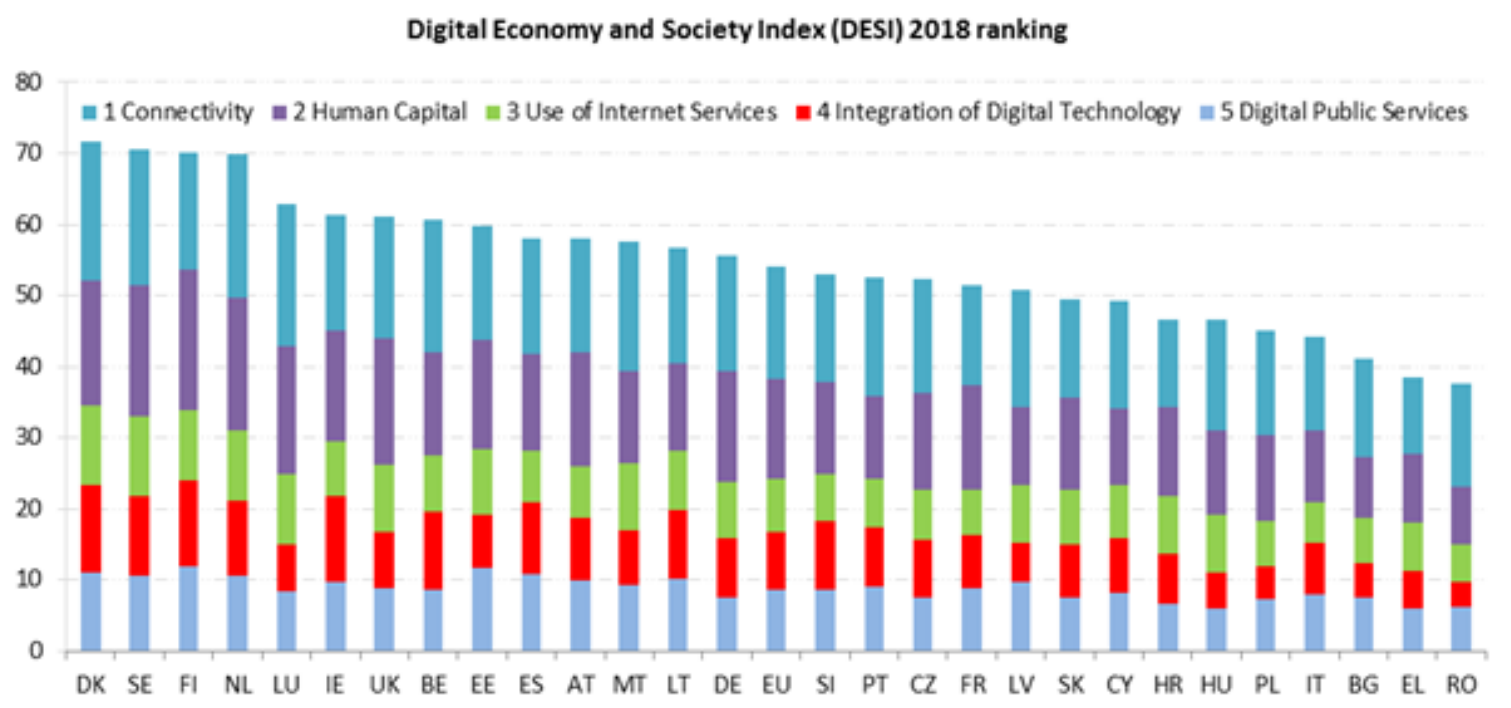


Con respecto a los cinco países con el PIB más importante de la Unión Europea, el Digital Economy and Society Index (DESI) 2018 rankingi, ubica a Alemania en el puesto 14, Reino Unido en el puesto 7, Francia en el 19, Italia en el 26 y finalmente España en el 10.

En el caso de Alemania que ocupa el puesto 14 del DESI 2018, progresando lentamente en el último año. Alemania lidera la asignación de espectro, facilitando el desarrollo de tecnologías móviles avanzadas en áreas rurales. Los alemanes tienen buenas habilidades digitales, no obstante, el potencial de la industria alemana corre el riesgo de verse obstaculizado por la escasez de profesionales de las TIC. Los usuarios alemanes de Internet son compradores en línea muy activos y las empresas alemanas están explotando las posibilidades que ofrece la economía digital. El mayor desafío del país en lo digital es mejorar la interacción en línea entre las autoridades públicas y los ciudadanos dado que solo el 19\% de la población es usuario de la administración electrónica (European Commission, 2018).

Reino Unido ocupa el puesto 7 del DESI 2018, se tiene que esta nación ha asignado el 69,2\% del espectro armonizado global para banda ancha, en comparación con la media de la UE del 67,7\% (European Comission, 2018). El Reino Unido ha alcanzado el $100 \%$ de cobertura rural de banda ancha fija, en comparación con la media de la UE del 93\%, en línea con su estrategia nacional de banda ancha (Council of the European Union, 2017).

Continuando con Francia, que ocupa el puesto 19 del DESI 2018, desde el año 2016, alcanzó una cobertura de banda ancha fija del 100\%, incluyendo las zonas rurales, superando la media de la UE del 98\%. La situación con respecto a las redes de alta velocidad es más compleja. Francia tiene una cobertura menor que la media de la UE para el acceso a la próxima generación de línea fija conocida como NGA ${ }^{\mathrm{ii}}$, lo cual supondrá un reto para esta nación en los próximos años (European Commission, 2016).

Por otro lado, tenemos a Italia que ocupa el puesto 26 del DESI 2018, mantiene una banda ancha fija estable, con una cobertura del 99\%. Con respecto a la cobertura NGA presenta un aumento significativo desde el año 2016 iii. Por lo tanto, el estado italiano tiene previsto aumentar significativamente la cobertura de banda ultra ancha durante el período 2016-2018 a través de la creación de una asociación estratégica con un proveedor alternativo de telecomunicaciones con el objetivo de acelerar la creación de la red de fibra (FTTH) en 29 ciudades con una inversión de 1.200 millones de euros (European Commission, 2016).

España que ocupa el puesto 10 del DESI 2018, sus redes de fibra desplegadas relativamente bien, España tiene las condiciones necesarias para garantizar que sus nacionales se beneficien de una economía digital innovadora y con garantía de futuro. En el contexto de una creciente tecnología de banda ancha ultrarrápida y la creciente agrupación de productos de comunicaciones electrónicas, la nueva configuración institucional que se está debatiendo actualmente en España será clave para abordar los problemas de precios de banda ancha fijos y móviles interrelacionados. El logro total del potencial socioeconómico de las redes de fibra y 5G dependerá de la creación de un 
entorno regulatorio y normativo que pueda abordar eficazmente los viejos problemas en el nuevo contexto tecnológico (European Commission, 2016).

Refiriéndose a los cinco países con el PIB menos importante de la Unión Europea, el Digital Economy and Society Index (DESI) 2018 ranking, ubica a Letonia en el puesto 20, Lituania en el 13, Eslovenia en el 16, Croacia en el 22 y finalmente Bulgaria en el 27.

Letonia que está ubicada en el puesto 20 del DESI 2018, tiene una cobertura de banda ancha fija básica relativamente baja (93\% en comparación con la media de la UE del 98\%), principalmente porque la cobertura en las zonas rurales es limitada. Lo sorprendente es que alrededor del $91 \%$ de los hogares tiene acceso de nueva generación (NGA). Resaltando que la cobertura rural fija de NGA creció significativamente en 10 puntos porcentuales hasta el $77 \%$ en 2016 , muy por encima de la media de la UE (European Commission, 2016).

Con respecto a Lituania ubicada en el puesto 13 del DESI 2018, la cobertura total de banda ancha fija alcanzó el $99 \%$ en 2016. La cobertura de banda ancha fija en las zonas rurales se mantuvo ligeramente por encima de la media de la UE (96\% frente al 93\%). Lituania es el líder de la UE en disponibilidad de $4 \mathrm{G}$ y penetración de fibra óptica. La cobertura 4G está significativamente por encima de la media de la UE ( $96 \%$ versus $84 \%$ ), ocupando el cuarto lugar entre los Estados miembros de la UE. La comunicación de banda ancha móvil de alta velocidad se está desarrollando en el país al aumentar la cobertura de la infraestructura 4G para la transmisión de datos a una velocidad ultra alta (European Commission, 2016).

Eslovenia ubicada en el puesto 16 del DESI 2018, ha logrado algunos avances en áreas rurales con cobertura fija de banda ancha y ahora está justo por debajo de la media de la UE (92\% versus 93\%). En términos de cobertura de acceso de próxima generación (NGA), Eslovenia continúa desempeñándose por encima de la media de la UE $(82 \%$ versus $76 \%$ ). Aunque se desempeña por encima de la media de la UE en la cobertura de NGA en áreas rurales ( $50 \%$ versus $40 \%$ ), Eslovenia sigue estando muy por debajo de la media de la UE en las suscripciones de banda ancha fija con más de $30 \mathrm{Mbps}$ ( $24 \%$ frente a una media de la UE del 37\%) (European Commission, 2016).

Croacia que está ubicada en el puesto 22 del ranking DESI 2018, en los últimos años no ha presentado mayores mejoras en la cobertura de la red de banda ancha fija en zonas urbanas y rurales ubicándose por debajo de la media de la UE que abarca el 93\%. Por lo tanto, la brecha entre las áreas urbanas y rurales son significativamente mayor en términos de cobertura de red de acceso de nueva generación (NGA). Si bien Croacia logró un buen progreso en general, logrando una cobertura total de NGA del $60 \%$, esta sigue por debajo de la media de la UE del 76\%. La cobertura de NGA en las zonas rurales se estanca en un $10 \%$, significativamente por debajo de la media de la UE del $40 \%$. Con un $67 \%$, la cobertura 4G de Croacia también es significativamente inferior a la media de la UE del 84\% (European Commission, 2016).

Finalmente, Bulgaria que ocupa el puesto 27 del ranking DESI 2018, en este país la cobertura total de las redes de banda ancha fija se mantiene sin cambios encontrándose por debajo de la media de la UE que ronda el 98\%. Además, la cobertura de banda ancha 
fija en las zonas rurales sigue siendo significativamente inferior a la media de la UE ( $81 \%$ versus 93\%). Las redes capaces de proporcionar al menos $30 \mathrm{Mbps}$ (acceso de nueva generación (NGA) están disponibles para casi las tres cuartas partes $(74 \%)$ de los hogares búlgaros, ligeramente por debajo de la media de la UE (76\%). En cuanto a la cobertura 4G, Bulgaria está rezagada con $66 \%$ frente a la media de la UE estimado en $84 \%$ (European Commission, 2016).

\section{Discusión}

Por lo tanto, esto significa que los cinco países de la Unión Europea con el PIB más alto cuyo promedio ascendió a 2.151.989 M.€ en el 2017, hacen un promedio de 15.2 puntos del RANKING DESI 2018, mientras que los cinco países de la Unión Europea con el PIB más bajo cuyo promedio ascendió a 42.219M.€ en el 2017, tienen un promedio de 19.6 puntos para el mismo periodo. También se podría agregar que, a pesar de tener una diferencia abismal en los promedios de PIB entre ambos grupos de países, tan solo la diferencia en el DESI 2018 es de 4,4. Con esto se quiere llegar a la conclusión que no es necesario ser un país con un PIB demasiado elevado para poder acortar la brecha digital. Un ejemplo de esto es Lituania, que, teniendo el segundo PIB más bajo de la Unión Europea durante el año 2017, se sitúa en el puesto 13 del ranking DESI 2018, con mejores resultados en la reducción de la brecha que Alemania, Francia e Italia (Ver tabla siguiente).

\begin{tabular}{|l|l|l|c|}
\hline & \multicolumn{2}{|c|}{ UNION EUROPEA 2017 } \\
\hline & PIB + ALTO & PAIS & RANKING DESI 2018 \\
\hline 1 & $3.263 .350 \mathrm{M} . €$ & Alemania & 14 \\
\hline 2 & $2.324 .293 \mathrm{M} . €$ & Reino Unido & 19 \\
\hline 3 & $2.291 .705 \mathrm{M} . €$ & Francia & 26 \\
\hline 4 & $1.716 .935 \mathrm{M} . €$ & Italia & 10 \\
\hline 5 & $1.163 .662 \mathrm{M} . €$ & España & 15.2 \\
\hline PROMEDIO & $2.151 .989 \mathrm{M} . €$ & & 20 \\
\hline & PIB + BAJO & & 13 \\
\hline 1 & $26.857 \mathrm{M} . €$ & Letonia & 16 \\
\hline 2 & $41.857 \mathrm{M} . €$ & Lituania & 22 \\
\hline 3 & $43.278 \mathrm{M} . €$ & Eslovenia & 27 \\
\hline 4 & $48.677 \mathrm{M} . €$ & Croacia & 19.6 \\
\hline 5 & $50.430 \mathrm{M} . €$ & Bulgaria & \\
\hline PROMEDIO & $42.219 \mathrm{M} . €$ & & \\
\hline
\end{tabular}

\section{Conclusiones}

En la Unión Europea lo digital está cobrando cada vez más importancia, a pesar de que algún grupo etario no se interese mucho en el tema, en particular los de la tercera edad. En 2017, se han presentado algunos logros en el tema de la reducción de la brecha digital, específicamente relacionado al tema de las economías digitales donde los países que destacan principalmente son Dinamarca, Suecia, Finlandia y los Países Bajos que han logrado un mayor avance en este rubro (European Commission, 2018).

La disminución de la brecha digital para los próximos años en la Unión Europea va a depender bastante de la prioridad que se le otorgue a esta problemática. Dado que la voluntad de disminuir los indicadores de la brecha digital no solo consiste en tener un presupuesto destinado a este rubro como consecuencia de un aumento sostenido en el 
PIB, sino también dependerá bastante de la voluntad política. Con respecto a los programas que la Unión Europea ha gestionado hasta el momento se puede afirmar que se ha producido un incremento significativo, dado que en el DESI 2017 la UE se ubicaba con un índice de 0,508; para el 2018 esta ha experimentado un aumento llegando a 0,54 (European Commission, 2018).

Esto se debe en parte a los programas "Europe 2020 Strategy" (2010), Gigabit Society by 2025 (2016) y WiFi4EU (2017) que han promovido de manera directa o indirecta la inclusión digital, reduciendo los índices de la brecha digital en la Unión Europea. No obstante se ha podido observar que la reducción de la brecha digital según el DESI 2018, y el de años anteriores ha mostrado que cada país de la Unión Europea lleva su propio ritmo de reducción de la brecha digital, donde no necesariamente el hecho de ser un país que se encuentre dentro de los primero cinco con el PIB más importante; como es el caso de Alemania, Reino Unido, Francia, Italia y España en el 2017, de ninguna manera es sinónimo de tener un avance significativo en la reducción de la brecha digital. Y, por otro lado, estar dentro de los cinco países con el PIB más bajo de este proceso de integración como Letonia, Lituania, Eslovenia, Croacia, Bulgaria para el mismo año, de ninguna manera es un impedimento para no poder presentar resultados satisfactorios en cuanto a la reducción de la brecha digital.

Sin embargo, un proceso de integración de la envergadura de la Unión Europea que pretende reducir la brecha digital en su territorio compuesta por 28 estados miembros, necesita una mayor precisión al momento de trabajar de manera conjunta para la reducción de este problema, al parecer cada país ha venido trabajando de manera independiente en función a la prioridad que se le asigne a este tema y en función a sus recursos. Para fundamentar esto, solo es necesario analizar el DESI de los años anteriores donde se puede apreciar tal disparidad.

Es casi seguro que la mayoría de los factores que van a jugar un rol fundamental en la disminución de la brecha digital para los próximos 5 años son los siguientes, desarrollo de infraestructura, economía digital, seguridad digital y los fondos privados para financiar programas de capacitación dirigidos a analfabetos digitales, concientización del buen uso del internet y un público objetivo estratégico y reducción progresiva de tarifas.

El desarrollo de infraestructura es vital para poder acortar la brecha digital entre las zonas urbanas y las grandes ciudades, especialmente en los países de la Unión Europea que pertenecen a Europa Central. A parte se puede continuar implementando el internet de banda ancha, ampliar el mercado de las telecomunicaciones, disminución de los precios de los servicios de telecomunicación, comparación de los aranceles con los países vecinos entre otras ventajas.

La economía digital será otro rubro que se va a fortalecer los próximos cinco años, lo que comprenderá la expansión del comercio electrónico, para lo cual la brecha digital tendría que haberse reducido para que se asegure un acceso equitativo a la conexión de Internet. Esto ayudaría a promover el Mercado Único Digital, con lo que se puede lograr un crecimiento sostenido y sustentable a través de la diversificación de nuevos productos 
y servicios. Para lograr esto se tendrá que trabajar en estimular las compras en líneas dado que en la actualidad dentro de la Unión Europea tan solo el 15\% de los consumidores compran en un país que no es el suyo. Por otro lado, y que es interesante señalar es que el 7\% de las pequeñas y medianas empresas venden bienes o servicios en el exterior de manera digital. Entonces, esto es lo que se va a tener que aprovechar ya que los medios digitales son las herramientas más propicias para desarrollar este Mercado Único Digital. Dicho mercado pretende unificar los mercados de los 28 países miembros en uno solo con la intención de generar 415, 000 millones de euros al año. No obstante, si se desea un despegue en este aspecto se requiere mejorar la estrategia actual de este mercado digital dando prioridad al acceso de consumidores y empresas a bienes y servicios digitales en toda Europa; crear un entorno propicio para el desarrollo de redes y servicios digitales innovadores y condiciones equitativas de competencia; maximizar el potencial de crecimiento de la economía digital (European Union, 2018).

La seguridad digital se tendrá que priorizar si es que verdaderamente se quiere reforzar la economía digital con miras a la consolidación de un Mercado Único Digital en la Unión Europea. Para lograr esto, se tendrá que dar importancia a la infraestructura computacional y a la información contenida o circulante especialmente la que fluye vía WIFI. En este punto se deberá de insistir en mejorar el marco legal de lo digital acompañado de protocolos de seguridad con la finalidad de minimizar la cyber criminalidad.

El sector privado a través de la responsabilidad social siempre será una buena opción al momento de desarrollar una sociedad más inclusiva por eso se deben de buscar iniciativas para que se financien capacitaciones dirigidas a empresas y analfabetos digitales que quieran adquirir conocimientos sobre las TIC's, donde se priorice el buen uso del internet. Además, se deberá identificar quien es el público objetivo potencialmente beneficiario donde se comprenda a personas que se encuentran en situación de vulnerabilidad.

Para poder lograr todo esto es necesario preservar la unidad política al interior de la Unión Europea, ya que sin esta no es posible que haya un panorama claro para los próximos cincos años (2018-2022). Lo más importante es trabajar de una manera pragmática centrándose como siempre lo ha hecho este proyecto de integración, es decir en el bienestar de sus ciudadanos. 


\section{Bibliografía}

Champain, V. (23 de junio de 2018). Les Echos. Obtenido de https://www.lesechos.fr/idees-debats/cercle/0301863960359-lutter-contre-leretour-de-la-fracture-numerique-2186606.php

Council of the European Union. (12 de mayo de 2017). Europa.Eu. Obtenido de http://data.consilium.europa.eu/doc/document/ST-9139-2017-ADD-61/en/pdf

Datos Macro. (2018). Datos Macro. Obtenido de https://datosmacro.expansion.com/pib

Euroepan Commission. (24 de julio de 2018). Europa.Eu. Obtenido de https://ec.europa.eu/digital-single-market/en/broadband-europe

European Comission. (2018). Obtenido de European Comission: http://ec.europa.eu/newsroom/dae/document.cfm?doc_id=44465

European Commission. (28 de mayo de 2014). Europa.Eu. Obtenido de https://ec.europa.eu/digital-single-market/en/news/eu-digital-divide-infographic

European Commission. (2015). Europa.Eu. Obtenido de https://ec.europa.eu/info/business-economy-euro/economic-and-fiscal-policycoordination/eu-economic-governance-monitoring-preventioncorrection/european-semester/framework/europe-2020-strategy_en

European Commission. (1 de diciembre de 2016). Euorpa.Eu. Obtenido de http://ec.europa.eu/newsroom/dae/document.cfm?doc_id=44451

European Commission. (1 de diciembre de 2016). Europa.Eu. Obtenido de ec.europa.eu/newsroom/document.cfm?doc_id $=44445$

European Commission. (1 de diciembre de 2016). Europa.Eu. Obtenido de http://ec.europa.eu/newsroom/dae/document.cfm?doc_id=44448

European Commission. (1 de diciembre de 2016). Europa.Eu. Obtenido de http://ec.europa.eu/newsroom/dae/document.cfm?doc_id=44458

European Commission. (1 de diciembre de 2016). Europa.Eu. Obtenido de http://ec.europa.eu/newsroom/dae/document.cfm?doc_id=44455

European Commission. (1 de diciembre de 2016). Europa.Eu. Obtenido de http://ec.europa.eu/newsroom/dae/document.cfm?doc_id=44461

European Commission. (1 de diciembre de 2016). Europa.Eu. Obtenido de http://ec.europa.eu/newsroom/dae/document.cfm?doc_id=44439

European Commission. (1 de diciembre de 2016). Europe.Eu. Obtenido de http://ec.europa.eu/newsroom/dae/document.cfm?doc_id=44449 
European Commission. (10 de mayo de 2017). Obtenido de European Commission: https://ec.europa.eu/digital-single-market/en/news/europes-digital-progressreport-2017-country-profiles-telecom-country-reports

European Commission. (03 de marzo de 2017). Europa.Eu. Obtenido de http://europa.eu/rapid/press-release_IP-17-347_fr.htm

European Commission. (03 de marzo de 2017). Europa.Eu. Obtenido de http://europa.eu/rapid/press-release_IP-17-347_fr.htm

European Commission. (2018). Europa.Eu. Obtenido de http://ec.europa.eu/information_society/newsroom/image/document/2018-20/bedesi_2018-country-profile_eng_199603A9-AB53-4E2103C0BEF3730537BA_52213.pdf

European Commission. (02 de agosto de 2018). Europa.Eu. Obtenido de https://ec.europa.eu/digital-single-market/en/europe-2020-strategy

European Commission. (08 de mayo de 2018). Europa.Eu. Obtenido de http://europa.eu/rapid/press-release_MEMO-18-3737_en.htm

European Commission. (26 de octubre de 2018). Europa.Eu. Obtenido de https://ec.europa.eu/digital-single-market/en/desi

European Commission. (19 de febrero de 2018). Europa.Eu. Obtenido de http://europa.eu/rapid/press-release_IP-17-347_fr.htm

European Commission. (2018). Europa.Eu. Obtenido de https://ec.europa.eu/information_society/newsroom/image/document/201820/de-desi2018-country-profile_eng_B43F6D80-F854-82CB6BF70068ACC73D74_52214.pdf

European Union. (junio de 2017). Europa.Eu. Obtenido de https://europa.eu/newsroom/highlights/special-coverage/end-roamingcharges_en

European Union. (04 de julio de 2018). Europa.Eu. Obtenido de https://europa.eu/european-union/about-eu/eu-in-brief_en

European Union. (24 de mayo de 2018). Europa.Eu. Obtenido de https://europa.eu/european-union/topics/digital-economy-society_fr

European Union. (06 de noviembre de 2018). Europa.Eu. Obtenido de https://europa.eu/european-union/about-eu/countries_es

Eurostat. (20 de septiembre de 2016). Eurostat. Obtenido de https://ec.europa.eu/eurostat/statisticsexplained/index.php/Glossary:Digital_divide

Plantard, P. (2011). Pour en finir avec la fracture numérique. Limousin: Editions YFP. 
Sander, A. (27 de junio de 2017). La Tribune. Obtenido de La Tribune: https://www.latribune.fr/opinions/tribunes/pour-une-europe-numerique741854.html

\section{Notas}

${ }^{\text {i }}$ El DESI 2018 es elaborado con cifras del año 2017

ii Next Generation Acces (Acceso de nueva generación)

Según el DESI 2017, en Italia la cobertura NGA aumentó significativamente del $41 \%$ de los hogares en 2015 al 72\% en 2016. Sin embargo, Italia sigue estando por debajo de la media de la UE (76\%). 